\title{
MYOELECTRIC ELBOW-WRIST-HAND ORTHOSIS WITH ACTIVE GRASP FOR PATIENTS WITH STROKE: A CASE SERIES
}

\author{
Eric Shoemaker,
}

Ability Prosthetics and Orthotics, Inc. 2005 Technology Parkway, Mechanicsburg, Pennsylvania, USA.

Email: eric.shoemaker@abilitypo.com

DOI: https://doi.org/10.33137/cpoj.v1i2.32022

\section{INTRODUCTION}

This case series presents prescription, evaluation, fitting and initial functional benefits of a myoelectric elbowwrist-hand orthosis with active grasp. Custom fit, myoelectric orthoses are now also being provided to patients with upper extremity paresis due conditions such as stroke, brachial plexus injury, spinal cord injury, multiple sclerosis and amyotrophic lateral sclerosis to enable them to self-initiate and control movement of a partially paretic limb using their own volitional myoelectric signals. A recent study of 18 chronic stroke participants demonstrated functional improvements on the Fugl-Meyer Impairment Scale (FM) and a battery of functional tasks with this device. ${ }^{1}$

METHODS

Patients are currently being evaluated by an interdisciplinary team at the Lebanon, PA outpatient VA clinic for the MyoPro 2 Motion $G$ device (Myomo Inc., Cambridge, MA). Two recent candidates who received the device were followed to evaluate the functional benefits they experienced. Patient 1: 67 year old male s/p CVA in 2013 affecting his right side. Patient 2: 61 year old male s/p CVA in 2014 affecting his right side. Both men were previously right hand dominant, both present with high motivation, and both have a strong family and care-giver support structure in place. During initial consultation, passive and active ROM were assessed as well as MMT. EMG signals were tested with the wrist flexors and extensors as well as biceps and triceps. Both men were able to generate and sustain an EMG signal for all 4 sites. Both men completed initial surveys with the Disabilities of the Arm Shoulder and Hand (DASH). Patient 1 scored 85 and patient 2 scored 67.5. A battery of functional tasks was also used to evaluate current deficits. The tasks included: lifting a laundry basket, bringing a spoon to the mouth, drink from a cup, turning on a light switch. Both patients were casted by a certified orthotist using fiberglass wrap from the level of the axilla and to include the hand in a neutral position. The cast was taken in one segments with assistance to capture the desired position at each joint. The custom MyoPro 2
Motion $\mathrm{G}$ was fabricated by a central fabricator, and the device was fit

within 3 weeks of casting. No adjustments were needed at the time of fitting. Programming was performed by the certified orthotist to optimize control of the device. Both patients began using Biceps Mode for elbow control and Voluntary Opening for grasp control. The software made it possible to change the control strategy of the device for better control of the assistive device for ADLs, because initially both patients struggled to control the elbow and grasp with independent and separate EMG signals from antagonist muscle groups. Both patients were referred to occupational therapy by a clinician trained in using the MyoPro.

\section{RESULTS}

Upon fitting of the MyoPro motion G, both patients were able to control flexion and extension of the elbow and open and close of the fingers. The patients still struggled to drink from a cup and bring a spoon to their mouths with the device but patient 2 was able to pick up a laundry basket and turn on a light switch with the device and was unable without. Active ROM was measured and showed an increase in both patients when wearing their MyoPro motion $\mathrm{G}$ devices. Patient 1 experienced an improvement in active ROM in elbow flexion of 10 degrees and elbow extension of 50 degrees with the MyoPro. Patient 2 showed a 35 deg improvement in elbow with the MyoPro. Active ROM with and without the MyoPro is depicted in Table 1.

Table 1: Active elbow range of motion with and without MyoPro

\begin{tabular}{|l|r|r|r|r|}
\hline & $\begin{array}{l}\text { Flexion } \\
\text { wo/MyoPro }\end{array}$ & $\begin{array}{l}\text { Flexion } \\
\text { w/MyoPro }\end{array}$ & \multicolumn{1}{l}{$\begin{array}{l}\text { Extension } \\
\text { wo/Myo Pro }\end{array}$} & $\begin{array}{l}\text { Extension } \\
\text { w/MyoPro }\end{array}$ \\
\hline Patient 1 & 110 & 120 & -50 & 0 \\
\hline Patient 2 & 120 & 120 & -35 & 0 \\
\hline
\end{tabular}

\section{CONCLUSION}

This case series highlights the unique orthotic intervention to restore active motion and independence with the upper extremity now available to patients with stroke. Initial fitting of the MyoPro 2 Motion G 
demonstrated increased active ROM of the both the elbow and the fingers for 3 jaw chuck grasp. Both patients showed an increase in active ROM with the MyoPro. These patients are expected to continue to follow up, and their progress with the MyoPro 2 Motion $\mathrm{G}$ will be documented throughout their recovery.

\section{SIGNIFICANCE}

Patients who've suffered a CVA have the potential to increase independence when performing certain activities of daily living. The ability to position the hand in space via elbow control and then grasp an object with the MyoPro can allow patients to perform bimanual activities such as lifting and carrying and laundry basket as was demonstrated here.

\section{REFERENCES}

1. Peters, HT. "Giving Them a Hand: Wearing a Myoelectric Elbow-Wrist-Hand Orthosis Reduces Upper Extremity Impairment in Chronic Stroke." Arch. of PM\&. pp 1821- 827, 2017. DOI:10.1016/j.apmr.2016.12.016

2. Takahashi KZ \& Stanhope SJ. ASB Annual Meeting, Boston, MA, USA, 2015.

\section{DISCLOSURE}

The authors are employed by Ability Prosthetics and Orthotics, Inc. and have no conflict of interest to report related to this case series. 